Poznańskie Studia Teologiczne 27(2013), s. 91-106.

Monika Adamczyk

Uniwersytet Pedagogiczny

Kraków

\title{
Edith Steins Weg zur Wahrheit
}

Edith Stein (1891-1942) war eine Schülerin und Assistentin von Edmund Husserl, die im Jahre 1922 zur katholischen Religion konvertierte und 11 Jahre später als Postulantin in den Kölner Karmel eintrat. Im Kloster hat sie ihre philosophischen Gedanken weiterentwickelt, wobei sie sich besonders mit dem Werk des hl. Thomas befasste, das sie schon vor ihrem Eintritt ins Kloster studiert hatte. Dort im Karmel ist ihr Hauptwerk Endliches und ewiges Sein ${ }^{1}$ entstanden.

Die Philosophie des hl. Thomas hat Stein von Anfang an deutlich in eine theozentrische Perspektive gerückt. So war die Philosophie für Edith Stein etwas, was sich zwischen dem Menschen und Gott ereignet, zwischen dem sterblichen und dem ewigen Seienden. Da die Phänomenologie von Edmund Husserl immer die Redlichkeit der Untersuchung ins Zentrum gestellt hat, war Steins an ihr geschultes und durch sie geformtes Denken streng und kritisch.

Das Ziel dieser Arbeit ist es, die Philosophie des hl. Thomas und die Phänomenologie Husserls im Kontext der Philosophie Edith Steins vorzustellen. Ich versuche darzustellen, wie eine Weltanschauung und eine Erkenntnismethode sich miteinander verknüpfen konnten. Auf der Grundlage von Ausschnitten aus Steins Schrift Endliches und ewiges Sein und aus dem Werk De veritate des hl. Thomas $^{2}$ (daraus die zwei ersten Quästionen), die die transzendentale Wahrheit betreffen, zeige ich, wie Stein methodisch gearbeitet hat. Diese Aufgabe war in einem doppelten Sinn eine schwierige, erstens aus dem Grund, dass eine ausführliche vergleichende Darstellung der Scholastik und der Phänomenologie im Rahmen dieser Arbeit nicht möglich ist, da sie deren Umfang weit überschreiten würde; zweitens deshalb, weil ich Edith Steins Übersetzung des De veritate verwenden wollte. Diese Übersetzung stellt eine Besonderheit dar: wie sie auch selbst gesagt hat, ist sie nämlich bereits eine Interpretation der Gedanken des Heiligen, die natürlich Spuren der phänomenologischen Methode in sich trägt ${ }^{3}$.

${ }^{1}$ E. Stein, Endliches und ewiges Sein, Freiburg 1950.

${ }^{2}$ Thomas von Aquin, Untersuchungen über die Wahrheit. Questiones disputatae de veritate 1 , Üb. E. Stein, ESGA 23, Freiburg - Basel - Wien 2008.

${ }^{3}$ Ihre Arbeit an De veritate kommentierte Stein selbst so: „Die Übersetzung scholastischer Text in einer Form, die auch für den modernen Menschen und Denker fassbar und schmackhaft ist, 
Nur durch die Analyse dieser Übersetzung des hl. Thomas ist es jedoch möglich zu zeigen, welche Rolle beide Denkarten in der Philosophie von Stein gespielt haben.

\section{Die allgemeine Charakteristik der Transcendentalia in Anlehnung an die Lehre des hl. Thomas aus dem ersten Artikel der ersten Quästion des De veritate}

Die Gedanken des hl. Thomas stellen den Versuch der Ableitung von Antworten auf grundlegende Fragen dar, die die menschliche Existenz so wie auch die Existenz Gottes betreffen. So wie frühere und zeitgenössische Denker baute Thomas hier auf den Lehren der Kirche auf, die er ordnete, verglich und prüfte. Seine Überlegungen führte er in Übereinstimmung mit der scholastischen Methode durch, die sich auf die drei Prinzipien legere, disputare und praedicare stützte. Auf diese Weise ist auch De veritate entstanden, das ursprünglich eine Vorlesungsreihe für Studenten darstellte, abgehalten in den Jahren 1256 bis 1259 in Paris.

Eine zentrale Aufgabe, die sich der hl. Thomas in De veritate gestellt hat, war die, eine vollständige und komplexe Beschreibung der Wahrheit in Übereinstimmung damit, was sie eigentlich ist, zu geben. Dementsprechend hat er verschiedene Dimensionen der Wahrheit betrachtet und untersucht, sich dabei unter anderem folgenden Themen widmend: die Erkenntnis des Menschen, die Erkenntnis Gottes und des Christus, der Glaube, die Gnade, die Erbsünde, das Gute usw. Jedoch musste er, um sein Ziel zu erreichen, eine sichere Grundlage finden. Deswegen hat er zu Beginn seiner Untersuchung ein Zurückführen zu dem, was für den Verstand klar ist und was dieser ohne Zweifel ergreifen kann, postuliert: „Wie man bei Beweisführungen auf irgendwelche Prinzipien zurückführen muss, die dem Verstand durch sich selbst bekannt sind, so bei der Erforschung dessen, was ein jedes ist; sonst würde es hier wie dort ins Unendliche fortgehen, und es würde alles Wissen und Erfassen der Dinge zunichte" ${ }^{\text {"4 }}$.

Die Grundlage des Wissens als solchen stellt für den hl. Thomas in erster Linie das Seiende dar. Da jedoch mit dem Seienden verschiedene andere Eigen-

ist erfahrungsgemäß eine sehr schwierige Sache. Man hat im Mittelalter den Übersetzer nicht umsonst interpres genannt, jede gute Übersetzung ist ein Kommentar, eine Interpretation. [...] Am besten wird eine solche Übersetzung gelingen, wenn derjenige, der sie herstellt, sowohl in der scholastischen Gedankenwelt gründlich zuhause ist, wie auch die Sprache der Gegenwartsphilosophie versteht“" (Vgl. ebd., Vorwort zur Ausgabe, S. 926).

${ }^{4}$ Thomas von Aquin, Untersuchungen über die Wahrheit. Questiones disputatae de veritate 1 , op. cit., S. 7; V. Ranff, Erstes Aufblitzen und ruhendes Schauen. Das Wahrheitsverständnis bei Edith Stein, „Jahrbuch für Religionsphilosophie“ (3) 2004, S. 195. 
schaften verbunden sind, die sich schon aus dem Grund, dass es allgemein existiert, auf das Seiende beziehen, rechnet Thomas diese ebenso diesem Fundament $\mathrm{zu}^{5}$. Diese notwendigen und universalen Charakteristika bezeichnen wir als Transcendentalia (lat. transcendere - übersteigen), sie werden von den individuellen Eigenschaften oder Eigentümlichkeiten, die zusätzlich jedem Seienden zukommen, unterschieden ${ }^{6}$. Die Rolle der Transcendentalia ist der Ausdruck dessen, was mit dem primären Begriff „das Seiende“ nicht umfasst wird. Als solche sind sie also keine äußere Natur, die etwas Neues zum Seienden hinzufügt, vielmehr stellen sie die Modi jedes Seienden dar. Beim hl. Thomas finden wir zwei Arten dieser Modi. Zu der ersten Art rechnen wir die Modi, die einem konkreten Seienden angehören, zur zweiten wiederum jene Modi, die jedem Seienden als solchem eignen. Weiter lassen sich die Modi differenzieren, indem man jene Modi, die zu jedem Seienden an sich gehören und jene, die zu jedem Seienden in der Ordnung, also in Bezug auf ein anderes Seiendes, gehören, voneinander unterscheiden. Zu der ersten Gruppe der Modi zählte der hl. Thomas, wie schon erwähnt, das ens (das Seiende), so wie auch res und unum, wobei die zwei ersten Bestimmungen das Seiende affirmativ bezeichnen (sie drücken aus, was das Seiende ist), während die dritte Bestimmung, also unum, es negativ charakterisiert (sie drückt aus, was das Seiende nicht ist). Zur zweiten Gruppe der Modi zählte der hl. Thomas folgende Bestimmungen: Aliquid, bonum und verum ${ }^{7}$.

Ens bezeichnet nach dem heiligen Thomas „das, was ist“, und hebt besonders das Seiende hervor - das, was existiert; res wiederum drückt das Seiende mit Rücksicht auf das, was es ist, d. h. seine Natur aus ${ }^{8}$. „Es lässt sich“, so Thomas,

aber keine affirmative, absolute Aussage finden, die in jedem Seienden angenommen werden könnte, außer seinem Seinsgehalt (Wesen, essentia), gemäß dem ihm das Sein (esse) zugesprochen wird; und so ist der Name Ding gegeben, das sich darin vom Seienden unterscheidet (nach Avicenna, Anfang der Metaphysik), dass das Seiende von der Aktualität des Seins hergenommen wird ( $a b$ actu essendi), der Name Ding (res) aber die Weisheit oder Wesenheit des Seienden (quidditatem sive essentiam entis) ausdrückt ${ }^{9}$.

${ }^{5}$ Vgl. Thomas von Aquin, Untersuchungen über die Wahrheit. Questiones disputatae de veritate 1 , op. cit., S. 7.

${ }^{6}$ Vgl. E. Stein, Ein nächtliches Gespräch. Was ist die Philosophie? Ein Gespräch zwischen Edmund Husserl und Thomas von Aquin, in: E. Stein, Erkenntnis und Glaube, Edith Stein Werke XV, Freiburg 1993, S. 35.

${ }^{7}$ Vgl. Thomas von Aquin, Untersuchungen über die Wahrheit. Questiones disputatae de veritate 1, op. cit., S. 7-8.

${ }^{8}$ Vgl. ebd, A. Wölfel, Das Problem der Wahrheit bei Anselm von Canterbury und Thomas von Aquin, Wien 2010, S. 23.

${ }^{9}$ Thomas von Aquin, Untersuchungen über die Wahrheit. Questiones disputatae de veritate 1 , op. cit., S. 8. 
Mit Unum bezeichnet der. hl Thomas die Unteilbarkeit des Seienden, was sich in dem Wort Eines ausdrückt ${ }^{10}$. Aliquid, das zu der zweiten Art der Transcendentalia gehört, bezieht sich auf das Seiende als Etwas, mit einem eigenen Was und Sein, durch das es etwas anderes (aliud quid) als das übrige Seiende ist $^{11}$. Um die zwei nächsten Bestimmungen des Seienden nachweisen zu können, suchte der hl. Thomas „etwas“, was aus seiner Natur heraus mit jedem Seienden in Übereinstimmung ist; und er fand dieses „Etwas“ schließlich in der Seele. Die Seele wird bei ihm durch verschiedene Vermögen (Fähigkeiten) charakterisiert, darunter das Vermögen, zu streben (Strebevermögen) und das intellektuelle Vermögen. Bonum hat der hl. Thomas als die Übereinstimmung des Seienden mit dem Streben definiert, verum wiederum drückt bei ihm die Übereinstimmung des Seienden mit dem Verstand aus ${ }^{12}$. Gerade diese letzte Bestimmung des Seienden wird uns am meisten interessieren. Wir werden versuchen zu verstehen, was diese Übereinstimmung des Seienden mit dem Verstand eigentlich bedeutet.

\section{2. „Was" und „wie" ist die transzendentale Wahrheit nach dem hl. Thomas?}

Der hl. Thomas hat mit Aristoteles behauptet, dass sich zwischen dem Seienden und dem Verstand eine Entsprechung befindet, d. h. das Seiende und der Verstand passen zusammen und existieren immer in Relation zueinander (die Wahrheit ist also bei Thomas von Aquin nicht nur ein Erkenntnisprozess). Diese Entsprechung zwischen Gegenstand und Verstand soll man als Übereinstimmung zwischen beiden Faktoren verstehen, was sich in der Definition adaequatio rei et intellectus ausdrückt. Diese Übereinstimmung ist nach dem hl. Thomas nur deswegen möglich, weil die Wahrheit zum Seienden die Gleichartigkeit bzw. Übe-

${ }^{10}$ Was genau der hl. Thomas mit dem Begriff ,die Unteilbarkeit des Seienden“ gemeint hat, zeigt uns Edith Stein deutlich in Endliches und Ewiges Sein. Die Unteilbarkeit des Seienden bedeutet nicht, dass das Seiende tatsächlich kein Teile besitzt, sondern es besteht aus verschiedenen Teilen, die endlich eine völlige und komplette Einheit darstellen „Die Einheit heißt transzendental, weil es sich um die Einheit des Seienden als solches handelt, d. h. Eines ist, was Ein Sein hat" und „Was hier mit «Einheit» bezeichnet ist, ist ein Letztes, was nicht mehr auf anderes zurückgeführt werden kann. Wenn es als Ungeteiltes bezeichnet wird, so ist das nur eine Erläuterung durch den Gegensatz, keine Zurückführung“(Vgl. ebd., E. Stein, Endliches und ewiges Sein, op. cit., S. 269).

${ }^{11} \mathrm{Vgl}$. Thomas von Aquin, Untersuchungen über die Wahrheit. Questiones disputatae de veritate 1 , op. cit., S. 8 .

${ }^{12}$ Das Wahre und das Gute bestimmen den Geist bei Edith Stein als Vollkommenheit gebend, weil er, wenn er sich auf sie zu bewegt, immer auf besondere Weise durch sie beeinflusst wird, da sie über ihr Sein (das Gute) oder ihren Gehalt (das Wahre) auf ihn einwirken, als ein Motor des Verfahrens der Aktualisierung der Potenzialität im menschlichen Dasein. Im Fall des Erkennens erreicht der Verstand seine Aktualität, im Fall des Strebens wiederum der Wille (Vgl. E. Stein, Endliches und ewiges Sein, op. cit., S. 290-292). 
reinstimmung mit dem erkennenden Geist hinzufügt. Das Ergebnis dieses Geschehens ist natürlich die Erkenntnis ${ }^{13}$.

Auf der Grundlage des oben Genannten hat Thomas von Aquin behauptet, dass wir die Wahrheit auf drei Weisen definieren können:

- in Anbetracht dessen, was das Fundament der Wahrheit darstellt, d. h. des Seienden (diese Definition hat schon der hl. Augustinus postuliert [,verum est id quod est"]);

- in Anbetracht dessen, was formal einen Wahrheitsbegriff darstellt (diese Definition hat Isaak postuliert, indem er sagte, dass die Wahrheit die Übereinstimmung der Sache mit der Erkenntnis sei [,,veritas est adaequatio rei et intellectus"] und der hl. Anselmus, der gesagt hat, dass die Wahrheit eine Richtigkeit darstelle, die nur mit dem Geist fassbar sei [, Veritas est rectuitudo sola mente perceptibilis"]);

- in Anbetracht der Folge der Wahrheit (die Definition von Hilarius: die Wahrheit offenbart und erhellt das Sein [,,quod verum est magnifestativum et decklarativum esse"] und des hl. Augustinus: Die Wahrheit ist es, wodurch das, was ist, sich zeigt und erscheint [,,Veritas est qua ostenditur id quod est" $])^{14}$.

Die Wahrheit in Bezug auf das Seiende zeichnet sich durch folgende Merkmale aus:

1. Die Wahrheit ist das, was ist, d.h. wahr ist das, was existiert und es existiert nur das Seiende, deswegen sagen wir, dass die Wahrheit mit dem Seienden austauschbar ist.

2. Das Seiende ist ohne die Wahrheit unfassbar, weil es auf keine andere Weise fassbar ist als im Bezug zum Verstand, dem es entspricht, und dieses Entsprechen nennen wir die Wahrheit.

3. Die Wahrheit ist keine äußere Eigenschaft des Seienden, sie umfasst es, indem sie ausdrückt, was nicht schon im Begriff des Seienden ausgedrückt wurde.

4. Das Seiende und die Wahrheit differenzieren sich nur gedanklich, weil im Bereich der Wahrheit etwas sein kann, was sich nicht im Bereich des Seienden befindet, jedoch nie so, dass sich etwas im Bereich des Seienden befindet, was nicht zugleich im Bereich der Wahrheit ist. Deswegen unterscheiden sie sich voneinander nicht dem Wesen nach.

5. Die Wahrheit fügt zum Seienden auch formal gar nichts hinzu, weil das Seiende so weit aufgefasst werden kann, dass es gleichzeitig das Seiende

${ }^{13} \mathrm{Vgl}$. Thomas von Aquin, Untersuchungen über die Wahrheit. Questiones disputatae de veritate 1, op. cit., S. 9; A. Wölfel, Das Problem der Wahrheit bei Anselm von Canterbury und Thomas von Aquin, op. cit., S. 24.

${ }^{14} \mathrm{Vgl}$. Thomas von Aquin, Untersuchungen über die Wahrheit. Questiones disputatae de veritate 1, op. cit., S. 9. 
und Nichtseiende umfasst. (Die Negation und Privation im Seienden ist in gewissem Sinne auch ein Seiendes, weil wir nur über einen Gegenstand, der ist, sprechen oder nachdenken können ${ }^{15}$.

6. Die Wahrheit liegt primär im Geist und sekundär im Seienden, weil die Wahrheit eine Übereinstimmung der Dinge mit dem erkennenden Geist darstellt und diese Übereinstimmung kann nur im Geist entstehen (das Ding kann nicht anders als wahr benannt werden, als dadurch, dass es mit dem Geist übereinstimmend ist ${ }^{16}$. Jedoch, schon Aristoteles hat die theoretische und praktische Vernunft voneinander unterschieden, indem er sagte, die praktische Vernunft besitze die Fähigkeit zur Erschaffung der Gegenstände, für die sie ein Maß darstellt. Die theoretische Vernunft wiederum, da sie im Bezug zu den natürlichen Dingen steht, ist passiv, nur „etwas“ annehmend von den Dingen, die dadurch sie messen (die Vernunft misst also nur die künstlichen Dinge und wird gleichzeitig selbst durch die natürlichen Dinge gemessen). Das letzte Maß für die Gegenstände stellt der Gottesverstand dar, in dem alle Dinge erschaffen wurden. So nennen wir das Ding wahr aus dem Grund, dass es mit beiden Verstandesarten übereinstimmt ${ }^{17}$. Im Bezug zur Gottesvernunft ist es wahr, wenn es das erfüllt, wozu es durch Gott erschaffen wurde, im Bezug zur menschlichen Vernunft wiederum ist ein Gegenstand wahr, sofern er ein richtiges Urteil über sich bewirkt ${ }^{18}$.

Die Gedanken des hl. Thomas aus der ersten und zweiten Quästion legen also dar, was die transzendentale Wahrheit ist und in welchem Bezug sie zum Gegen-

${ }^{15}$ Vgl. ebd., S. 9-12.

${ }^{16}$ Vgl. ebd., S. 13.

${ }^{17}$ Vgl. A. Wölfel, Das Problem der Wahrheit bei Anselm von Canterbury und Thomas von Aquin, op. cit., S. 26-27.

${ }^{18}$ Die Wahrheit, die so zu beurteilen ist, nennt Stein in Endliches und ewiges Sein die ontologische Wahrheit. Die ontologische Wahrheit besteht dann, wenn ein Ding alles besitzt, was zu seinem Wesen gehören soll (die Wahrheit wird hier als die Echtheit verstanden). Die ontologische Wahrheit beurteilt der Mensch auf dem Grund seiner Idee von dem Ding, die für ihn wie „ein Vorbild“ ist, vorstellend, wie das Ding eigentlich sein soll. Jedoch ist die menschliche Idee, an der wir einen Gegenstand messen, kein letztes Maß, weil sie selbst wahr oder falsch sein kann. Ein letztes Kriterium der Echtheit von etwas kann nur die göttliche Idee (das echtes Vorbild der Dinge in der Gottesvernunft) darstellen, die identisch mit der sog. reinen Form des Gegenstands ist. Diese reine Form hat Gott jedem Seienden geschenkt, als er es erschuf. Deswegen kann man noch über die Wesenswahrheit, die Stein als die Übereinstimmung der Wesensform eines realen Dings (die Wesensform zeigt, wie ein Gegenstand als existierender ist) mit seiner reinen Form. Diese Wahrheit ist letztendlich die Grundlage dafür, dass ein Ding wahr sein kann. Davon müssen wir noch die logische Wahrheit differenzieren, die mit den menschlichen Urteilskräften verbunden ist. In einem Urteil ergreifen wir den Sachverhalt von etwas und wenn dieser Sachverhalt wirklich besteht, dann ist auch unser Urteil wahr. Natürlich hat alle diese Arten von Wahrheit bereits der hl. Thomas postuliert (E. Stein, Endliches und ewiges Sein, op. cit., S. 275-279; A. Wölfel, Das Problem der Wahrheit bei Anselm von Canterbury und Thomas von Aquin, op. cit., S. 29). 
stand steht. Dieses Ziel konnte Thomas durch die Anwendung der historisch-vergleichenden und analytischen Methode erreichen, sich dabei zunächst auf die Gedanken früherer Denker stützend, um dann das, was in den einzelnen Begriffen enthalten ist, zu untersuchen. Auf diese Weise hat er ein Kompendium des Wissens nicht nur von der Wahrheit erschaffen, sondern ein Kompendium des Wissens vom Menschen als solchem und in seinem Bezug zu Gott; und sein Werk ist bis heute ein Rätsel und eine Herausforderung für jeden Philosophen.

\section{3. „Was“ und „wie“ ist die transzendentale Wahrheit nach Edith Stein?}

Die Transcendentalia hat Edith Stein so wie der hl. Thomas als Bestimmungen des Seienden als solches definiert, die das, was zum inneren Wesen jedes Seienden gehört, d. h. seine formalen Charakteristika, ausdrücken. Nach dem heiligen Thomas hat sie hier: ens, res, unum, aliquid, bonum und verum erwähnt ${ }^{19}$.

Um zu einem Verständnis der transzendentalen Wahrheit zu gelangen, fragte Stein: Was ist die Wahrheit als solche?, und begann ihre Untersuchungen mit der Analyse der Beziehung zwischen dem Gegenstand und dem Wissen. Hier benutzt Stein einen Begriff, den auch der hl. Thomas im zweiten Teil von De veritate gebraucht hat, nämlich die reine gedankliche Beziehung. Dieser Begriff bezeichnet die Unabhängigkeit des Gegenstands gegenüber dem Wissen, da er immer, ungeachtet dessen, ob jemand ihn begreift und versteht oder nicht, bleibt, was er ist (die menschliche Erkenntnis des Gegenstands trägt gar nichts zu seinem Inhalt oder seinem formalen Aufbau bei, sie verändert ihn nie). Diese Beziehung, obgleich bilateral, beweist die Abhängigkeit des Wissens, weil dieses erstens ohne den Gegenstand nicht existieren kann (es verdankt ihm seine Wirklichkeit, d. h. den Durchgang von der seelischen Fähigkeit zum tätigen Akt) und zweitens sein eigenes „Was“ dem Gegenstand verdankt. Diese Beziehung wiederum ist als die reale Beziehung bezeichnet worden ${ }^{20}$. Hier stellt Edith Stein jedoch die Frage, was es eigentlich bedeutet, dass die Beziehung des Wissens zum Gegenstand real ist? Ist es so, dass sie ebenso real wie irgendein Gegenstand ist? Sie antwortet: Wenn wir unter der Beziehung des Wissens zum Gegenstand ihre Richtung, ihre Intention (Stein nannte dies „das Meinen“ des Gegenstands) verstehen, dann

${ }^{19} \mathrm{Zu}$ diesen transzendentale Bestimmungen rechnen wir manchmal auch das Schöne, was auch Stein erwähnt: ,(Das Schöne hat mit beiden etwas Gemeinsames [mit dem Gute und dem Wahren - M.A.] es ist das Seiende als Gegenstand des Wohlgefallens, das in dem Erlebnis der objektiven Übereinstimmung des Seienden mit dem Erkennen - in der Ordnung ihres Aufbaus - begründet ist)" (E. Stein, Endliches und ewiges Sein, op. cit., S. 264; Vgl. Thomas von Aquin, Untersuchungen über die Wahrheit. Questiones disputatae de veritate 1, op. cit., S. 7-8).

${ }^{20} \mathrm{Vgl}$. E. Stein, Endliches und ewiges Sein, op. cit., S. 273; Thomas von Aquin, Untersuchungen über die Wahrheit. Questiones disputatae de veritate 1, op. cit., S. 17. 
ist diese Beziehung so real wie jeder Gegenstand. „Dieses Meinen“, erwähnt Stein, „(die Intention) ist ja ein wesentliches Bestandstück des Wissenserlebnisses und hat an dessen Sein Anteil. Etwas Entsprechendes ist im Gegenstand nicht aufzuweisen" ${ }^{\text {221 }}$.

Die Beziehung des Wissens zum Gegenstand ist jedoch noch keine Übereinstimmung, die wir als Wahrheit benennen könnten, obwohl sie unentbehrlich für die Existenz der Wahrheit ist, weil diese sich auf ihrer Basis konstituiert. Dies ist jedoch nur dann möglich, wenn das Wissen und der Gegenstand die zwei folgenden Anforderungen erfüllen: 1. Der mutmaßliche Gegenstand muss immer real existieren (der Gegenstand, der als wirklicher gemeint wird, muss als solcher existieren). 2. Der Gehalt des Wissens muss mit der realen Gestalt des Gegenstands übereinstimmen (der Gegenstand als gemeinter muss genau der sein, der in der Wirklichkeit existiert). Nur dann, wenn Deckungsgleichheit zwischen diesen beiden Faktoren besteht, entsteht die Übereinstimmung, die nicht beiden implizit ist, sondern sich zwischen ihnen ereignet. Stein versteht also die Wahrheit als Beziehung - als Relation ${ }^{22}$.

Indem sie den Begriff der Wesenswahrheit und der ontologischen Wahrheit in der Philosophie des hl. Thomas analysierte, hat Stein erkannt, dass die transzendentale Wahrheit keine von ihnen ist, obwohl sie mit ihnen in einer engen Beziehung steht. Die transzendentale Wahrheit findet Stein im Bezug zum Sinn des Gegenstandes (das entsprechende scholastische Wort ist intelligibile), der in einen erkennenden Geist eindringen kann und diesem erlaubt, ihn zu erfassen ${ }^{23}$. Deswegen suchte Stein jene Faktoren, die die Bedingungen dieses Erfassens darstellen. Letztendlich hat Stein diese im Offenbarsein des menschlichen Geistes und der Zugänglichkeit / dem Offenbarsein jedes Dinges gefunden, die in deren Natur eingeschrieben sind (das „Offenbarsein“ gehört sowohl zu jedem Teil des Dinges wie auch zu ihm als Ganzheit, aber besonders umfasst es das Sein des Seienden $)^{24}$. Stein schreibt:

Im Verhältnis zu dem, was ,irgendein Seiendes“ in sich ist, ist sein Offenbarsein oder seine Zuordnung zum Geist etwas Neues. Versteht man allerdings unter „,das Seiende“ „alles Seiende“, und zwar als Ganzes: die Gesamtheit alles dessen, was ist; dann ist auch die Ordnung dieses Ganzen darin eingeschlossen. Es gehört zum Seienden als Ganzem, dass es ein geordnetes Ganzes ist: dass jedes einzelne Seiende darin seinen Platz und seine geregelten Beziehungen zu allem anderen hat; die Ordnung ist

\footnotetext{
${ }^{21}$ E. Stein, Endliches und ewiges Sein, op. cit., S. 274; Vgl. E. Husserl, Ideen zu einer reinen Phänomenologie und phänomenologische Philosophie. Allgemeine Einführung in die reine Phänomenologie 1, Tübingen 1980, S. 179-188.

${ }^{22} \mathrm{Vgl}$. E. Stein, Endliches und ewiges Sein, op. cit., S. 274.

${ }^{23}$ Vgl. ebd., S. 276; Thomas von Aquin, Untersuchungen über die Wahrheit. Questiones disputatae de veritate 1, op. cit., S. 13.

${ }^{24}$ Vgl. E. Stein, Endliches und ewiges Sein, op. cit., S. 276.
} 
ein Teil des (so verstandenen) Seienden, und das Offenbarsein oder die Zuordnung zum Geist, die uns mit der transzendentalen Wahrheit gleichbedeutend ist, ist ein Teil dieser Ordnung ${ }^{25}$.

Die Anwendung des Begriffes „transzendental“ ist nach Stein gerechtfertigt, weil die Zuordnung zum Geist nicht zu einer Gattung des Seienden gehört, sondern zu jedem Seienden (zu jedem Einzelnen oder zum Ganzen). Jedoch ist der Geist eine besondere Gattung des Seienden, weil er für alles, was existiert, offen ist. Indem er sich damit beschäftigt, erfüllt er sein eigenes Seiendes. Auf diese Weise ist der Geist eine Gattung des Seienden, der alles andere Seiende in bestimmter Weise zugeordnet ist; und die Wahrheit stellt eine besondere Form der Zuordnung alles Seienden zum offenen Geist dar ${ }^{26}$.

\section{Die Brücke in der Geisteswissenschaft: Die phänomenologische Methode in der Verknüpfung mit dem thomistischen Denken}

Die Phänomenologie wurde und wird bis heute unterschiedlich interpretiert. Die Einen verstehen sie als ein System, innerhalb dessen wir vielfältige Probleme lösen können. Die Anderen begreifen sie nur als eine Methode, die hilfreich zur Untersuchung verschiedener Bereiche der Realität ist. Diese zweite Interpretation kann man, wie ich glaube, als übereinstimmend mit den Gedanken des Begründers der Phänomenologie selbst, Edmund Husserl, lesen, obwohl dies in der Perspektive des Gesamtbauwerks, welches sein phänomenologischer Idealismus darstellt, anders scheinen mag. In Wirklichkeit war für Husserl die Phänomenologie nämlich schon vom Beginn seines philosophischen Suchens an ein methodisches Fundament, auf dessen Grundlage eine strenge Metaphysik entstehen sollte. Im Brief an Peter Wust, den Urheber des berühmten Werks Die Auferstehung der Metaphysik, hat Husserl diese Idee skizziert: „,...] meine Philosophie von vornherein nicht etwas anderes war und sein wollte, als der Weg zu einer radikal echten, ehrlich begründeten, streng wissenschaftlichen Metaphysik [...]. Schon in meiner Antrittsvorlesung 1887 trat ich für eine neue wissenschaftliche Metaphysik ein“"27; was letztendlich zeigt, dass sie beide ein sehr ähnliches wissenschaftliches Ziel verband. Der Gegenstand der Bestrebungen von Husserl war die absolute Wahrheit, in deren Licht der Mensch alles, wie es eigentlich ist, erkennen kann. Gerade auf diese Weise hat auch Edith Stein die Phänomenologie

${ }^{25}$ Ebd., S. 276-277.

${ }^{26}$ Vgl. ebd., S. 277.

${ }^{27}$ P. Wust, Gesammelte Werke, Bd. VIII, Münster 1967, S. 30; zitiert nach: E. Avé-Lallemant, Edmund Husserl zu Metaphysik und Religion, in: Husserl in Halle: Spurensuche im Anfang der Phänomenologie, hrsg. H.M. Gerlach, H. Rainer Sepp, Frankfurt am Main 1994, S. 87. 
verstanden. Für sie war die Phänomenologie eine Methode der Lebensbetrachtung, die dank der einzelnen Schritte, die der Philosoph ausführen soll, die Möglichkeit zu einer tieferen Anschauung der Wirklichkeit gibt. Als solche hat sie Stein begriffen und war damit im Grunde genommen nicht besonders weit vom Thomismus entfernt, obschon es natürlich zwischen den beiden Strömungen mehr oder weniger große Unterschiede gibt. Diese Überzeugung hat sie auf den Seiten des Aufsatzes ausgedrückt, der ein fiktives Gespräch zwischen Husserl und dem hl. Thomas schildert, unter dem Titel: Ein nächtliches Gespräch. Was ist die Philosophie? Ein Gespräch zwischen Edmund Husserl und Thomas von Aquin.

Die Gedanken des hl. Thomas und Edmund Husserls sind vor allem durch konzeptuelle Aspekte, wie die Gestalt der Philosophie und ihr eigentliches Ziel, verbunden. Für den hl. Thomas sollte Philosophie eine strenge Wissenschaft darstellen - eine Grundlage für die menschliche Suche nach der Wahrheit, die eigentlich Gott selbst ist. In jeder philosophischen Betrachtung sollte bei dem älteren Philosophen die ratio der materiellen Dinge entdeckt werden, was nur durch die Betrachtung einer Reihe materieller Gegenstände zu erreichen ist, die später miteinander verglichen werden, um sie am Ende dem Abstraktionsprozess zu unterwerfen $^{28}$. Bei Husserl soll die Philosophie auch eine exakte Wissenschaft sein, die sich ebenso nach der Wahrheit richtet. Die Teilnahme an ihr ist nur dann möglich, wenn der Mensch sich in seiner Erkenntnis jeweils nach dem Wesen des Gegenstands richtet und besondere methodische Schritte vollzieht ${ }^{29}$. Nach Stein gibt es jedoch zwischen diesen beiden Ansichten einen fundamentalen Unterschied, nämlich sei die Wahrheit, von der Husserl spricht, nicht Gott, weil sein Forschungsweg rein egozentrisch und nicht theozentrisch $\operatorname{sei}^{30}$. Heute wissen wir, dass Edith Stein sich geirrt hat, denn sowohl der hl. Thomas wie auch Edmund Husserl suchten in ihren Forschungen einzig Gott selbst. Die Phänomenologie war nämlich nach Husserls Ansicht ein Weg zu Gott ${ }^{31}$, der - wie Roman Ingar-

\footnotetext{
${ }^{28}$ Vgl. E. Stein, Ein nächtliches Gespräch. Was ist die Philosophie? Ein Gespräch zwischen Edmund Husserl und Thomas von Aquin, op. cit., S. 39-42.

${ }^{29} \mathrm{Vgl}$. E. Husserl, Die Idee der Phänomenologie, herg. P. Janssen, Hamburg 1986, S. 23-24, 29-32, 55-58.

${ }^{30}$ Vgl. E. Stein, Ein nächtliches Gespräch. Was ist die Philosophie? Ein Gespräch zwischen Edmund Husserl und Thomas von Aquin, op. cit., S. 7.

${ }^{31} \mathrm{Im}$ Brief an Arnold Metzger, wo Husserl sich an die Wirkung des Studiums der Bibel auf seine Person erinnert, hat er das erste Mal auf diese Weise die Philosophie definiert. So hat er darüber geschrieben: „Denn die gewaltige Wirkung des Neuen Testament auf den 23jährigen lief doch in dem Triebe aus, mittelst einer strenger philosophischen Wissenschaft den Weg zu Gott und zu einem wahrhaften Leben zu finden“. Die gleiche Ansicht hat Husserl auch im Brief an Roman Ingarden von 1924 und im Gespräch mit Schwester Jägerschmied ausgedrückt (E. Husserl, Brief an Arnold Metzger vom 4. September 1919, in: A. Metzger, Phänomenologie der Revolution, Frankfurt am Main 1979, S. 106; vgl. E. Husserl, Brief an Roman Ingarden vom Ende Dezember 1924,
} 
den gesagt hat - das eigentliche Zentrum seines Denkens darstellte ${ }^{32}$. Wie ich vermute, hat Stein das nicht erkannt, weil alle Schriften von Husserl, in denen das Gottesthema eine wichtige Rolle spielt, erst nach ihrem Tod veröffentlicht wurden $^{33}$. Außerdem müssen wir uns dessen bewusst sein, dass Husserls Weg zu Gott ein besonderer war. Im Gespräch mit Schwester Adelgundis Jägerschmid hat er darüber gesagt:

Das Leben des Menschen ist nichts anderes als ein Weg zu Gott. Ich versuche, dieses Ziel ohne theologische Beweise, Methoden und Stützpunkte zu erreichen, nämlich zu Gott ohne Gott zu gelangen. Ich musste Gott gleichsam aus meinem wissenschaftlichen Dasein eliminieren, um den Menschen einem Weg zu Gott bahnen, die nicht wie Sie die Sicherheit des Glaubens durch die Kirche haben ${ }^{34}$.

Diese Aufgabe hat, wie Husserl meinte, eine absolute Fundierung der ganzen Philosophie erfordert und gerade ihr hat er sich ganz hingegeben und sich deswegen erst am Ende seines Lebens kurz mit dem Gottesthema an sich beschäftigt ${ }^{35}$.

in: Edmund Husserl, Briefwechsel, Husserliana Dokumente, Bd. III/3, hrsg. K. Schuhmann, Dordrecht - Boston - London 1994, S. 224; A. Jägerschmid, Gespräche mit Edmund Husserl, in: E. Stein, Wege zur inneren Stille, Aschaffenberg 1987, S. 217.

${ }^{32}$ In seiner Rede anläßlich des hundertsten Jahrestags von Husserls Geburt hat Ingarden gesagt: „Letztendlich suchte Husserl einen Weg zu Gott als dem höchsten und absoluten Verstand, in welchem er seine eigene Vervollkommnung und endgültigen Frieden zu finden hoffte und auf den er eine Teleologie des reinen Bewusstseins aufbauen könne" (R. Ingarden, Z badań nad filozofia wspótczesna [Aus den Untersuchungen zur Gegenwartsphilosophie], Warszawa 1963, S. 627).

${ }^{33}$ Es geht hier um: Die Krisis der europäischen Wissenschaften und die transzendentale Phänomenologie (1936), Erfahrung und Urteil. Untersuchungen zur Genealogie der Logik (1939), Cartesianische Meditationen (1950) und Formale und transzendentale Logik. Versuch einer Kritik der logischen Vernunft (1974). Außer diese Abhandlungen gibt es viele Schriften von Husserl, die mit diesem Thema verbunden sind, die bis heute noch nicht veröffentlicht sind wie z. B. Ethisches Leben. Theologie - Wissenschaft (1924-1927), Absolutes Bewusstsein. Metaphysisch (1908/1909), Teleologie (1930), Universale Ethik. Wissenschaft und Leben. Wissenschaft und Ethik - Theorie und Praxis, mythische und Wissenschaftliche Weltbetrachtung, Philosophisches Leben (1931), Metaphysik und Teleologie (1930-1934), Liebe, Teleologie, Ich. Das personale Ich und die individuelle Eigenheit (1920-1921, 1934-1935), Enthüllungsgang der universalen Teleologie als Gang der gesamten Konstitution von den Anfängen an (1933-1934).

${ }^{34}$ A. Jägerschmid, Gespräche mit Edmund Husserl, op. cit., S. 219.

${ }^{35}$ Zeitgenössische Erforscher der Gedanken von Husserl haben Zugang zu zahlreichen Schriften zum Gottesthema in der Husserlschen Phänomenologie. Wichtige Quelle stellen z. B. die Artikel von S. Strasser, Der Gott des Monadenalls. Gedanken zum Gottesproblem in der Spätphilosophie Husserls (Hildesheim 1958) und History, Theology, and Got in the Philosophy of Husserl (Dordrecht 1979), L. Landgrebe, Husserls phänomenologischer Zugangsweg zu den Problem der Religion (Marburg 1986), W. Keilbach, Zu Husserls phänomenologischen Gottesbegriff (Paderborn 1976), E. Avé-Lallemant, Edmund Husserl zu Metaphysik und Religion (Frankfurt am Main 1994) und die Bücher A. Ales Bello, Husserl. Sul problema di Dio (Roma 1985), M. García Baró, Ensayos sobre Lo Absoluto (Madrid 1993), Lee Chun Lo, Die Gottesauffassung in Husserls Phänomenologie (Frankfurt am Main 2008) dar. 
Da die weitere Ähnlichkeiten so wie Unterschiede zwischen beiden Strömungen wiederum vor der inhaltlichen Grundlage deutlich werden, schlage ich die Hinwendung zur Steinschen Analyse der Wahrheit vor, die am besten zu ihrer Vorstellung dienen kann.

Die Methode der Untersuchung des Materials, die Stein in dieser Analyse ausgewählt hat, ist die genaue Beschreibung des Subjekts, Schritt für Schritt, um die Sache als solche zu erfassen. Diese Beschreibung stellt einen wichtigen Teil der phänomenologischen Methode dar. Die Anwendung dieser Beschreibung fordert vom Philosophen - kurz gesagt - die Verwandlung seiner erkennenden Einstellung zur Welt und sich selbst. Der Philosoph soll alle wissenschaftlichen Thesen, eigenen Vorurteile, mitgebrachtes Wissen, Überzeugungen usw. einklammern. Dieses methodische Verhalten nennen wir die phänomenologische Reduktion. Eine wichtige Voraussetzung dieser Reduktion stellt bei Husserl das Ausklammern des Seins der Welt dar (Husserl war der Überzeugung, dass die reale Existenz der Welt zur Erkenntnis des Gegenstands nicht nötig ist, weil alles sich im Bewusstsein als ein Korrelat von etwas präsentiert, an sich schon ein Gegenstand des Bewusstseins), das das reflektierende Zurückführen zu sich selbst, zum Selbstbewusstsein, das ein Feld des Gewissheit darstellt, ermöglicht. Im Bewusstsein präsentieren sich verschiedene Gegenstände, die das absolute Ich genau so, wie sie sind, erfasst ${ }^{36}$.

Es zeigt sich, dass Stein die phänomenologische Methode angewandt hat, die jedoch für sie eine Art der Elimination aller Faktoren, die mit dem zu erforschenden Gegenstand nicht verbunden sind, war ${ }^{37}$. Stein führte ihre Untersuchungen so durch, dass sie sich maximal auf den Gegenstand ihrer Forschungen konzentrierte, zurückkehrend zum Wesen des Seienden, um später sein Sosein hervorzubringen. Mit dieser Situation haben wir es zu tun, wenn sie z. B. sagt, dass die Wesenswahrheit und die ontologische Wahrheit noch nicht das sind, was sie eigentlich sucht; und erst recht, wenn sie den Gegenstand ihrer Untersuchungen bereits erfasst hat und, unter Zugrundlegung dessen, wann und wie sie besteht, die transzendentale Wahrheit beschreibt ${ }^{38}$. Umso mehr richtete sich Steins Suche allerdings an der Frage „wie?“ aus, gleich jener Husserls, jedoch setzten ihre

${ }^{36}$ Vgl. E. Husserl, Die Idee der Phänomenologie, op. cit., S. 53-56; E. Husserl, Ideen zu einer reinen Phänomenologie und phänomenologische Philosophie. Allgemeine Einführung in die reine Phänomenologie 1, op. cit., S. 53-57, 108-120.

${ }^{37}$ Es ist erwähnenswert, dass auch der hl. Thomas ein Zurückführen postulierte, nämlich das Zurückführen zu den grundlegenden Begriffen, die die Vernunft anwendet, um die fundamentalen Dimensionen der Wirklichkeit zu ergreifen. Auf diese Weise hat auch der hl. Thomas alles, was hinderlich zur Erkenntnis des Gegenstands ist, aus dem Raum der Untersuchungen ausgegrenzt. (Vgl. Thomas von Aquin, Untersuchungen über die Wahrheit. Questiones disputatae de veritate 1, op. cit., S. 7; V. Ranff, Erstes Aufblitzen und ruhendes Schauen. Das Wahrheitsverständnis bei Edith Stein, op. cit., S. 159).

${ }^{38}$ Vgl. E. Stein, Endliches und ewiges Sein, op. cit., S. 275-277. 
Forschungen schon die Existenz des Gegenstands wie auch der Welt voraus. Betrachten wir ihre Untersuchungen zum Phänomen des Meinens, wo sie behauptet, dass die Wahrheit dort möglich ist, wo der Gegenstand, der als wirklicher gemeint wird, als solcher existiert und wenn er genau so gemeint ist, wie er in der Wirklichkeit existiert ${ }^{39}$. Hier folgt Stein eigentlich dem hl. Thomas, der die realistische Methode der Erkenntnis postuliert hat, die bei Stein notwendig zur sogenannten eidetischen Erkenntnis (die Erkenntnis des Wesens der Dinge, die Husserl postuliert hat) ist. In dem fiktiven Gespräch mit Husserl sagt Thomas von Aquin:

Für dieses praktische, aber auch für das theoretische Ziel einer möglichst umfassenden Welterkenntnis war es nötig, empirische und eidetische Kenntnisse zu verwenden. Was Glaube zum Weltbild beisteuert, ist ja vorwiegend Tatsachenwissen, wenn auch ein Wissen von anderer Valenz als das aus sinnlicher Erfahrung. Der Unterschied war mir indessen durchaus geläufig. Ich sprach von dem, was den Dingen ,an sich“ ihrer „Essenz“ nach zukommt - wesenhaft, wie Sie sagen - und dem, was ihnen ,akzidentell“" zukommt, vermöge der Konstellation, in die sie durch den praktischen Verlauf des Geschehens hineingeraten $\sin ^{40}$.

Das Objekt der Erkenntnis war bei Husserl ein absolutes $\mathrm{Ich}^{41}$, das die Fähigkeit zur vollen Einsicht in den Gegenstand - genauer in das Wesen des Gegenstands - besitzt ${ }^{42}$. Das Ziel des Strebens von Edith Stein war auch das Wesen des Gegenstandes; wie z. B. in unserem Forschungsmaterial der Sinn der transzendentalen Wahrheit, die für sie letztendlich die Übereinstimmung des erkennenden Geistes mit dem Seienden war, welche auf der Grundlage des Sinns jedes einzelnen Gegenstands (der Gegenstand ist seiner Natur nach zugänglich/ /offen) und des Sinns des menschlichen Geists (der Geist ist auch seiner Natur gemäß offen für den Gegenstand) existiert ${ }^{43}$. Unter dem Einfluss des hl. Thomas hat Stein jedoch nicht wie Husserl an die Möglichkeit der vollen Anschauung des Wesens geglaubt, sie erkannte eine klare Grenze des Wissens und kam so zum Gedanken der Gestalt der Gnade.

\footnotetext{
${ }^{39}$ Vgl. ebd., S. 274.

${ }^{40}$ E. Stein, Ein nächtliches Gespräch. Was ist die Philosophie? Ein Gespräch zwischen Edmund Husserl und Thomas von Aquin, op. cit., S. 35.

${ }^{41}$ Für Stein war eine fundamentale Dimension des Menschen die Person, die die Quelle und das Zentrum aller erfüllenden Akte war, deswegen hat sie die Gestalt des Menschen ganz anders als Husserl verstanden. Ihre eigene Ansicht hat sie vor allem in: Der Aufbau der menschlichen Person. Vorlesung zur philosophischen Anthropologie, ESGA 14, Freiburg - Basel - Wien 2004; Bildung und Entfaltung der Individualität: Beiträge zum christlichen Erziehungsauftrag, ESGA16, Freiburg - Basel - Wien 2004; Was ist der Mensch?: theologische Anthropologie, ESGA 15, Freiburg - Basel - Wien 2005, ausgedrückt.

${ }^{42} \mathrm{Vgl}$. E. Husserl, Ideen zu einer reinen Phänomenologie und phänomenologische Philosophie. Allgemeine Einführung in die reine Phänomenologie 1, op. cit., S. 94-100.

${ }^{43}$ Vgl. E. Stein, Endliches und ewiges Sein, op. cit., S. 276.
} 
Der hl. Thomas hat ebenso wie Husserl das Wesen des Gegenstandes (ratio der materiellen Dinge) gesucht, postuliert jedoch, dass dazu nicht nur die Fähigkeiten der Vernunft (die theoretische Vernunft, die Stein auch die übernatürliche nennt) notwendig sind, sondern auch der Glaube und die Offenbarung. Die Offenbarung ist unentbehrlich zur echten Erkenntnis, denn obwohl die menschliche Vernunft eine Anschauung in die inneren Strukturen des Gegenstandes hat und verschiedene erste Prinzipien erkennen kann, steht sie letztendlich immer vor dem Geheimnis, das Gott und die göttliche Kreatur darstellen ${ }^{44}$. Der hl. Thomas spricht zu Husserl:

Er ist [der Geist - M. A.] jetzt auf der Wanderschaft dieses Lebens begriffen, und wird einst ans Ziel, ins himmlische Vaterland kommen. Am Ziel wird er alles umfassen, was ihm fassbar ist (nicht alle Abgründe der göttlichen Wahrheit, die allein Gott ganz umfasst), und zwar wird er dies alles in einer einzigen Intuition schauen. Manches von dem, was er dann schauen wird, soviel ihm nötig ist, um den Weg zum Ziel nicht zu verfehlen - wird ihm durch Offenbarung mitgeteilt; er fasst es im Glauben, der auf der irdischen Pilgerschaft ein zweiter Weg, neben der natürlichen Erkenntnis ist, um Wissen zu erlangen. [...] In allgemeinen ist es so, dass nur das Glaubenssache ist, was prinzipiell unserer irdischer Erkenntnis entzogen ist. Doch ist auch manches durch Offenbarung mitgeteilt, was auf dem Weg der Erkenntnis nur von wenigen oder nicht mit genügender Sicherheit erkannt werden könnte ${ }^{45}$.

Am Ende meiner Überlegungen möchte ich noch eine Neuerung in den Gedanken über die transzendentalen Wahrheit erwähnen, die Edith Stein zu ihrer Interpretation beigetragen hat ${ }^{46}$. Ich meine hier das Konzept der Intentionalität, das ein Fundament des phänomenologischen Denkens darstellt, welches Stein in ihren Überlegungen über die Beziehung zwischen dem Wissen und dem Gegenstand angewandt hat. Bei Edmund Husserl besteht zwischen dem Erkennenden und dem Gegenstand eine besondere Beziehung, weil das menschliche Bewusstsein immer ein Bewusstsein von etwas ist (wir denken an etwas, wir zweifeln an etwas, wir fragen nach etwas usw.). Das bedeutet, dass das Bewusstsein sich immer mit einer Intention auf etwas richtet - es ist immer auf angemessene Weise eingestellt. Diesen Erkenntnisvollzug nennt Husserl Noesis. Der Gegenstand, der bis jetzt ganz passiv war, kann sich im Bewusstsein des Subjekts präsentieren, welches jetzt ihn passiv ergreift. Den Gegenstand, der sich im menschlichen Bewusstsein präsentiert, nennt Husserl Noema ${ }^{47}$. Stein, unter Berück-

${ }^{44}$ Vgl. V. Ranff, Erstes Aufblitzen und ruhendes Schauen. Das Wahrheitsverständnis bei Edith Stein, op. cit., S. 194.

${ }^{45}$ E. Stein, Ein nächtliches Gespräch. Was ist die Philosophie? Ein Gespräch zwischen Edmund Husserl und Thomas von Aquin, op. cit., S. 24-25.

${ }^{46}$ Das Konzept der Intentionalität hat Husserls Lehrer, Franz Brentano, postuliert, der sich kurz mit dem Thomismus beschäftigt hat. Vermutlich hat er Spuren davon gerade in dieser Philosophie gefunden (Vgl. ebd., S. 22).

${ }^{47} \mathrm{Vgl}$. E. Husserl, Ideen zu einer reinen Phänomenologie und phänomenologische Philosophie. Allgemeine Einführung in die reine Phänomenologie 1, op. cit., S. 179-188. 
sichtigung der Intention (also dem Meinen des Gegenstands), beweist die Realität der Beziehung zwischen dem Wissen und dem Gegenstand, die doch die Wahrheit bedeutet. Erinnern wir uns noch einmal daran, was sie genau gesagt hat: „Dieses Meinen (die Intention) ist ja ein wesentliches Bestandstück des Wissenserlebnisses und hat an dessen Sein Anteil. Etwas Entsprechendes ist im Gegenstand nicht aufzuweisen" $" 48$.

Das Ziel von Husserls Untersuchungen war Gott selbst, deswegen hat er in einem Gespräch mit Schwester Adelgundis gesagt: „Was die Kirchen wollen, will ich auch: die Menschheit hinführen zur Aeternitas. Meine Aufgabe ist es, dies durch die Philosophie zu versuchen. [...] Das ist mein Lebenswerk ${ }^{* 49}$. Die Erreichung dieses Ziels war für ihn nur möglich (und darüber mögen wir staunen) dank der Verbindung der Phänomenologie und der Lehre der Kirche. Ein Stück weiter im selben Gespräch können wir lesen:

[...] dass gerade meine Phänomenologie, und nur sie die Philosophie ist, die die Kirche brauchen kann, weil sie mit dem Thomismus zusammenführt und die thomistische Philosophie weiterführt. Warum hält die Kirche starr [nur - M. A.] am Thomismus fest? Wenn die Kirche lebendig ist, muss sie sich auch in der Phänomenologie weiterentwickeln. Das Wort Gottes ist immer dasselbe, ewige. Aber die philosophische Interpretation ist abhängig von den jeweiligen lebendigen Menschen einer Zeit. Sie ist also relativ ${ }^{50}$.

Wie ich glaube, hat Edith Stein, später Schwester Teresia Benedikta vom Kreuz dieses Ziel von Husserls Streben erreicht und so die Aufgabe der Phänomenologie erfüllt, die sie mit ihrem Leben bestätigt hat.

$* * * * * * *$

Sowohl der hl. Thomas wie auch Edmund Husserl haben daran geglaubt, dass die Erreichung einer strengen und exakten Philosophie möglich ist, die uns schließlich der Wahrheit näher bringt. Der Weg zu ihr war in beider Philosophien mehr oder weniger ähnlich.

Edith Stein hat mit ihren eigenen Gedanken versucht, eine Brücke zwischen ihnen aufzubauen, wobei die Phänomenologie nur die Methode gibt, ohne die jedoch eine echte Untersuchung der Wirklichkeit nicht möglich wäre. Die thomistische Philosophie wiederum war für Edith Stein, wie wir gesehen haben, eine Quelle von Gedanken, die sie jedoch nicht naiv übernommen hat, sondern deren einzelne Thesen sie methodisch untersucht, expliziert und geprüft hat. Dann hat sie aus diesem Denken geschöpft, was sie als das Beste erkannte und dies mit ihren eigenen Ideen untermauert und weiterentwickelt.

\footnotetext{
${ }^{48}$ E. Stein, Endliches und ewiges Sein, op. cit., S. 274.

${ }^{49}$ A. Jägerschmid, Gespräche mit Edmund Husserl, op. cit., S. 213.

${ }^{50}$ Ebd., S. 216-217.
} 
Auf der Grundlage der Steinschen Analyse der Wahrheit wollte ich zeigen, wie sie eigentlich die Phänomenologie und den Thomismus verbunden und so letztendlich ihren eigenen Weg zur Wahrheit gefunden hat. Ich denke nämlich, dass Stein nicht nur die Philosophie von Edmund Husserl und dem hl. Thomas fortgeführt, sondern ihre eigene entwickelt hat, d. h. die Philosophie der Edith Stein, bekannt auch als die hl. Benedikta vom Kreuz.

\section{Ways to the Truth of Edith Stein}

\section{Summary}

On her intellectual journey to the truth, which she after all defined as God himself, Edith Stein had managed to combine two philosophical approaches such as phenomenology and thomism. Former approach was considered by her as a method of describing the reality, which after fulfilling certain steps of methodology, could allow one to reveal the essence of things. The latter one, was perceived by her as a wide and versatile source of ideas obtained not only from epistemology and ontology, but also human philosophy and theology. These ideas were adopted by Stein and developed by her own sophisticated implications. By comparing Thomas Aquinas' analysis on understanding the truths, contained in the first two parts of Disputed Questions on Truth (Questiones Disputatae de veritate), with her reflections on the same aspect included in Finite and Eternal Being: An Attempt to an Ascent to the Meaning of Being, Saint Teresa Benedicta of the Cross discloses her explanation of synthesising these two philosophical schools, with her reasons and consequences of doing so.

\section{Edyty Stein droga do prawdy}

\section{Streszczenie}

Poszukując drogi do Prawdy, którą okazał się ostatecznie sam Bóg, Edyta Stein połączyła ze sobą dwa kierunki myślenia: fenomenologię oraz tomizm. Pierwszy z nich był dla niej metodą oglądu rzeczywistości, dzięki której przy spełnieniu określonych metodologicznych kroków możliwe było ujęcie istoty rzeczy. Drugi z kolei był źródłem wielorakich idei, zarówno z zakresu epistemologii, ontologii, jak również filozofii człowieka czy teologii, które to E. Stein częściowo przejęła oraz na swój własny, oryginalny sposób rozwinęła. Porównując analizy prawdy autorstwa św. Tomasza, zamieszczone w dwóch pierwszych kwestiach De veritate z rozważaniami Stein na ten sam temat zawartymi w Bycie skończonym a bycie wiecznym, autorka szkicuje sposób, w jaki filozof oraz święta uzgodniła ze sobą oba kierunki, omawiając jednocześnie przyczyny oraz skutki takiego zabiegu.

\section{Slowa kluczowe}

fenomenologia, tomizm, transcendentalia, prawda, istota, poznanie

\section{Keywords}

phenomenology, thomism, transcendent matters, truth, being, knowing 